\title{
Energy balance for biodiesel production processes using microbial oil and scum
}

Lalit R.

Sravan Kumar

Xiaolei Zhang

R.D. Tyagi ${ }^{\mathrm{a}, *}$

Rd.tyagi@ete.inrs.ca

a INRS Eau, Terre et Environnement, 490, rue de la Couronne, Québec G1K 9A9, Canada

${ }^{\mathbf{b}}$ School of Civil and Environmental Engineering, Harbin Institute of Technology (Shenzhen), Shenzhen, Guangdong 518055, China

*Corresponding author.

Abstract

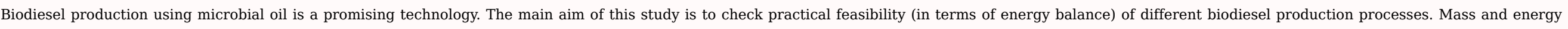

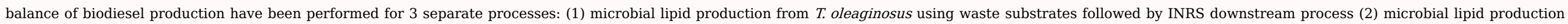

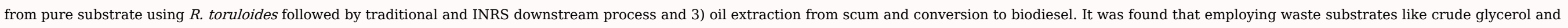

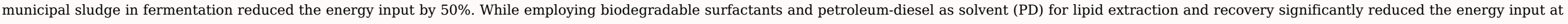
cell wall disruption step. Biodiesel production from scum is a two-step process which is fast and energetically favorable.

Keywords: Energy balance; Biodiesel production; Waste sources; Scum

\section{Introduction}

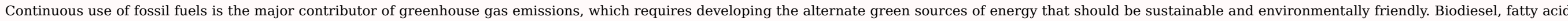

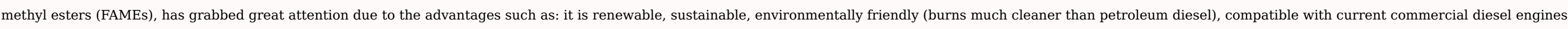

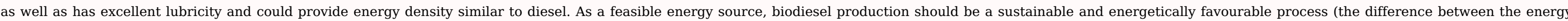
output and the energy input is positive) (Thamsiriroj and Murphy, 2011; Zhang et al., 2013).

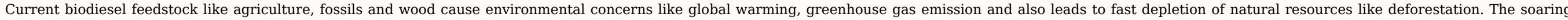

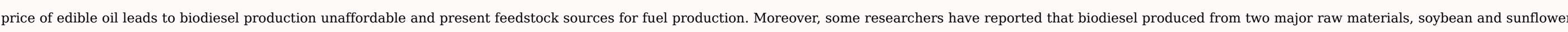

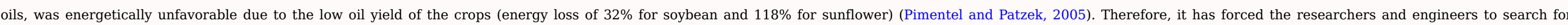

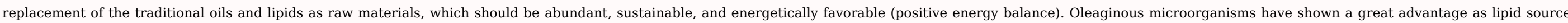

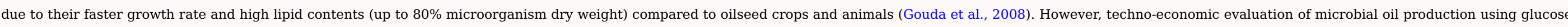

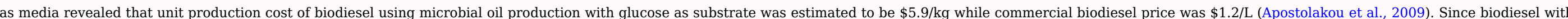
microbial oil technology with glucose as substrate is an expensive process, alternatives for inexpensive sources should be looked upon.

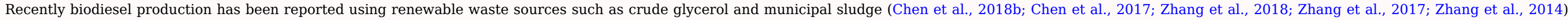

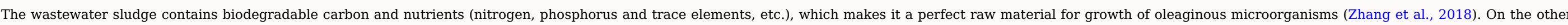




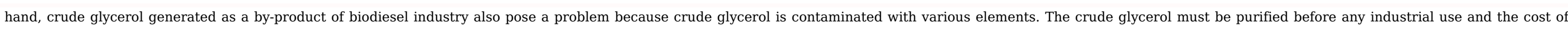

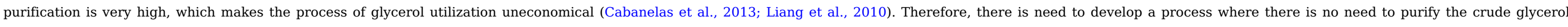

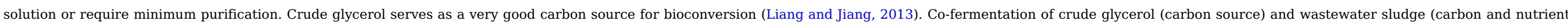

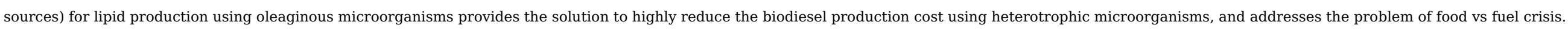

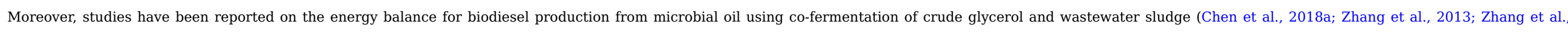

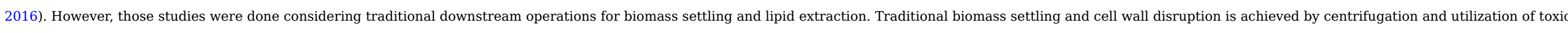

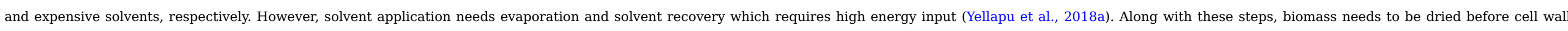

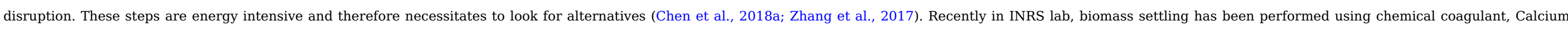

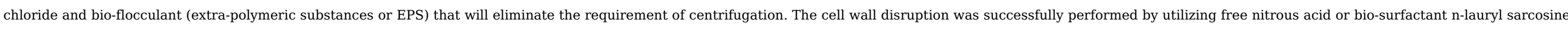

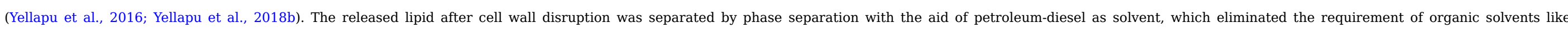

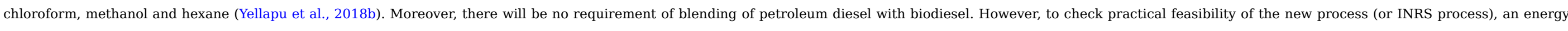

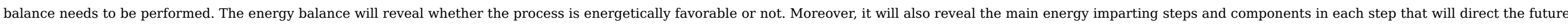
research for microbial oil-based biodiesel_:

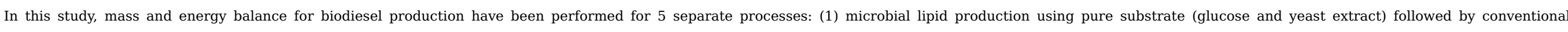

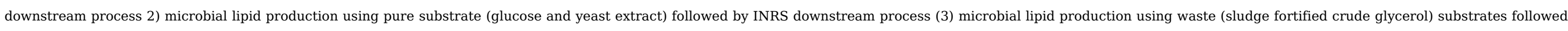
by INRS downstream process 4) microbial lipid production using crude glycerol followed by INRS downstream process and 5) direct oil extraction from scum and conversion to biodiesel.

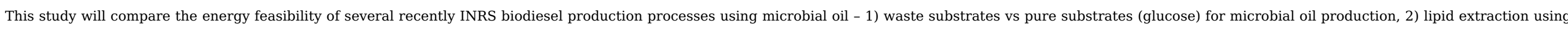

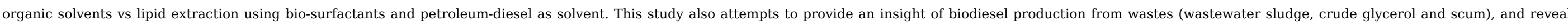
which process of biodiesel production could be energetically feasible.

\section{Methodology}

\subsection{Calculation basics}

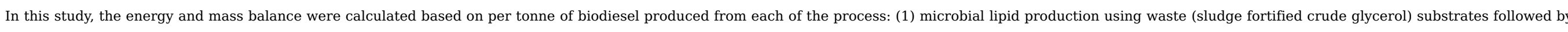

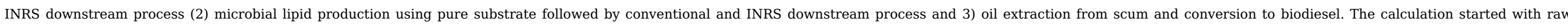

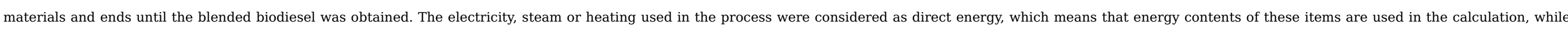

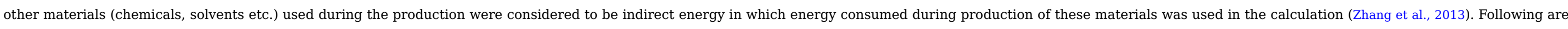
the important energy terms used during making energy balance calculations:

Energy input: Sum of all energy inputs at every process step

Energy credit: Internal energy present in co-products

Net energy input: The difference between energy input and energy credit

Energy balance: Energy contained in produced biodiesel after subtracting the net energy input

Energy ratio: The ration between energy output and net energy input

For an energetically favourable process, net energy balance across the complete process should be positive or energy ratio (output/input) should be greater than 1 .

\subsection{Process description}




\subsubsection{Microbial lipid production using pure substrate (glucose and yeast extract) followed by conventional downstream process}

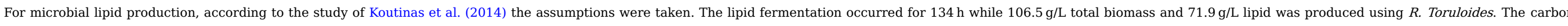

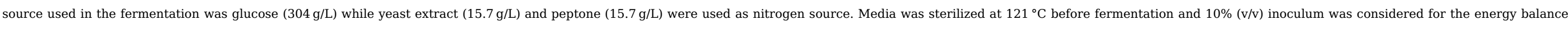

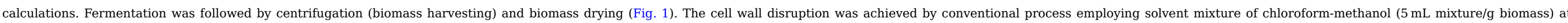

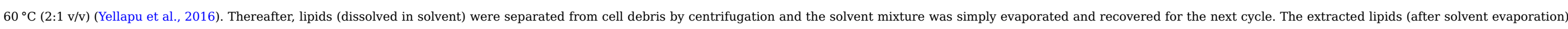

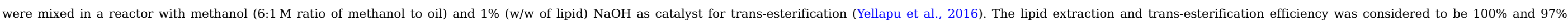

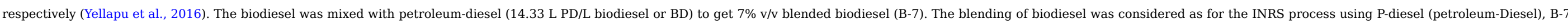
is the final product. In the conventional process for microbial oil biodiesel, 6 steps were involved to get blended biodiesel.

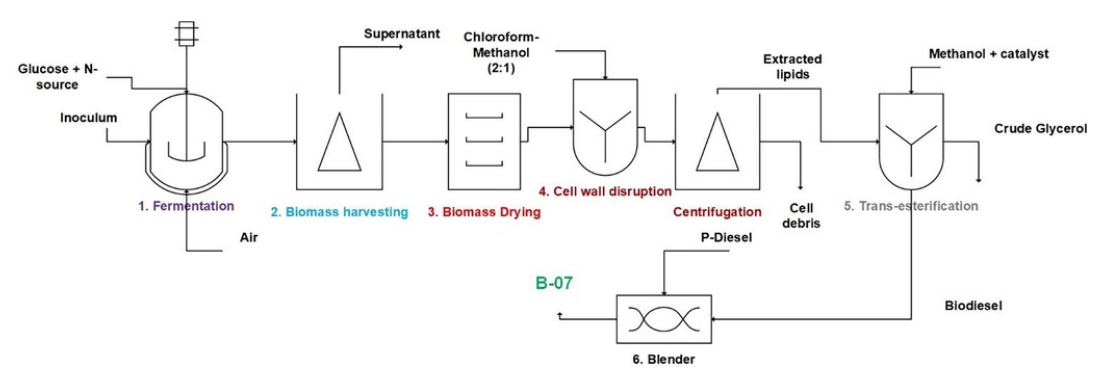

Fig. 1 Blended biodiesel production (B-7) using microbial oil with pure substrate (glucose) followed by conventional downstream process.

\subsubsection{Microbial lipid production using pure substrate (glucose and yeast extract) followed by INRS downstream process}

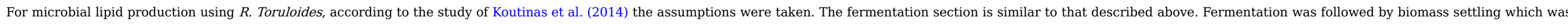

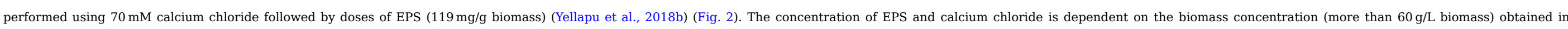

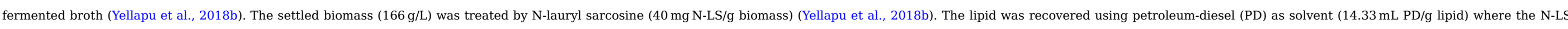

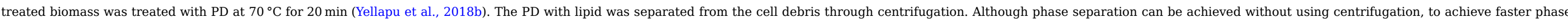

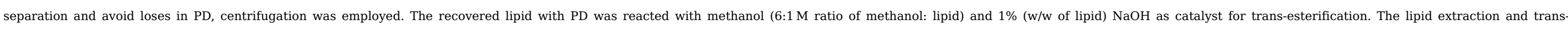

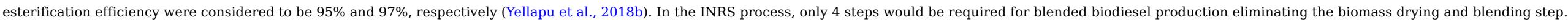

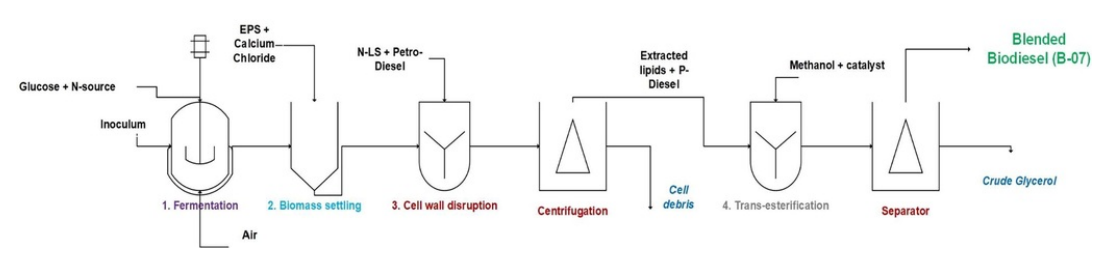

Fig. 2 Blended biodiesel production (B-7) using microbial oil with pure substrate (glucose) followed by INRS downstream process.

\subsubsection{Microbial lipid production using waste substrates (sludge fortified with glycerol) followed by INRS downstream process}

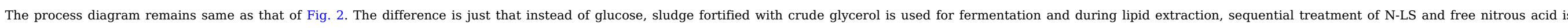

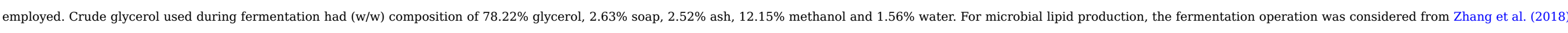

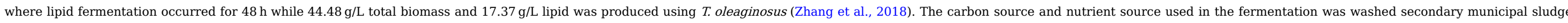

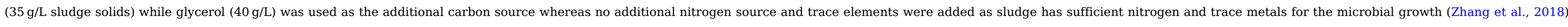

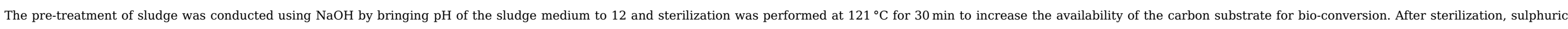




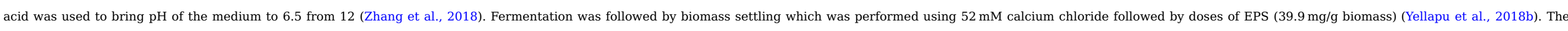

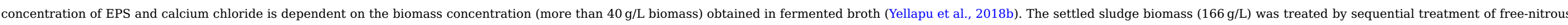

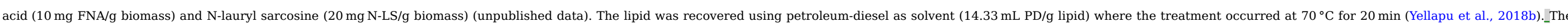

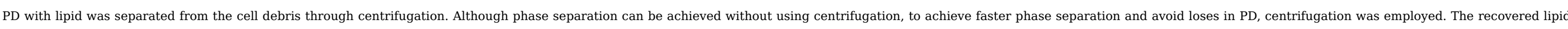

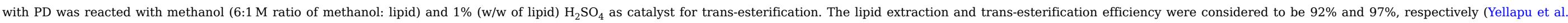
2018b).

\subsubsection{Microbial lipid production using crude glycerol media followed by INRS downstream process}

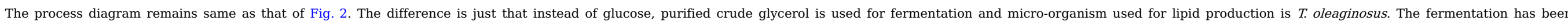

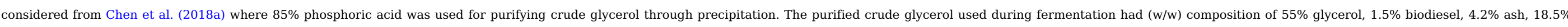

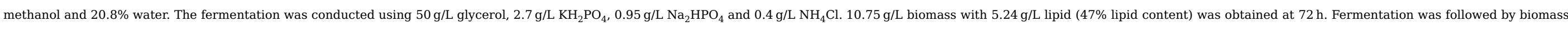

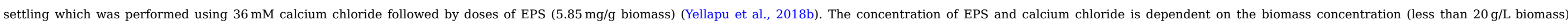
obtained in fermented broth (Yellapu et al., 2018b). The lipid extraction and trans-esterification were performed similar as described in Section 2.2.2.

\subsubsection{Direct oil extraction from scum and conversion to biodiesel}

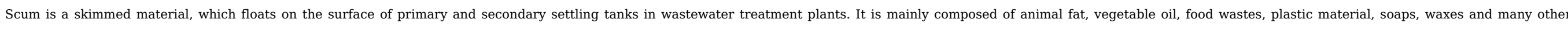

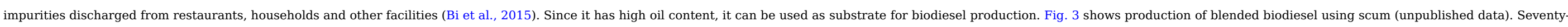

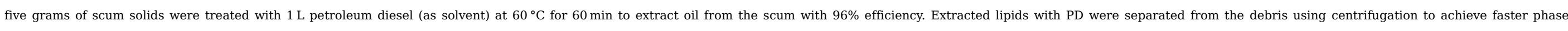

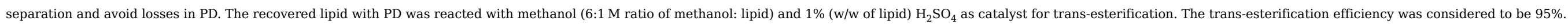

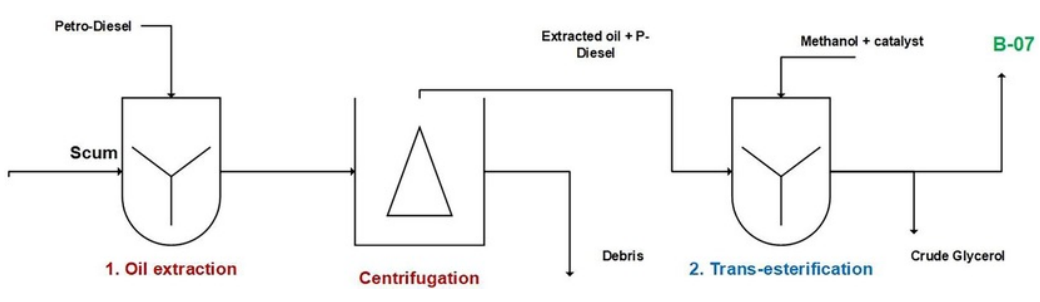

Fig. 3 Blended biodiesel production (B-7) using scum.

\subsubsection{Trans-esterification of recovered lipids}

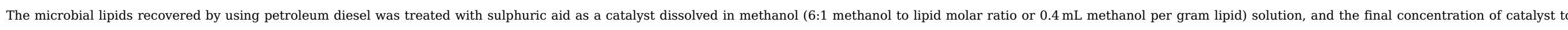

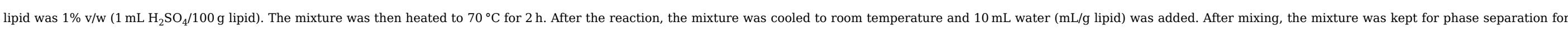

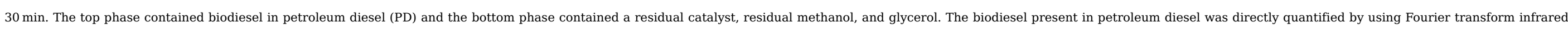
spectrometer (FT-IR) to determine the percentage of biodiesel present in the petroleum diesel. The characterization of biodiesel produced from scum and microbial oil is provided in Table 1.

Table 1 Characterization of biodiesel produced from microbial oil and scum.

\begin{tabular}{|c|c|c|}
\hline Fatty acid & Biodiesel obtained from microbial oil & Biodiesel obtained from scum \\
\hline C14:0 (\%) & 1.51 & 7 \\
\hline C15:0 (\%) & 3.23 & - \\
\hline C16:0 (\%) & 27.89 & 35 \\
\hline
\end{tabular}




\begin{tabular}{|l|l|}
\hline C16:1 (\%) & 20.11 \\
\hline C17:0 (\%) & 1.71 \\
\hline C18:0 (\%) & 11.33 \\
\hline C18:1 (\%) & 21.82 \\
\hline C18:2 (\%) & 2.42 \\
\hline C18:3 (\%) & 1.03 \\
\hline C20:1 (\%) & 1.31 \\
\hline
\end{tabular}

\section{Results and discussion}

\subsection{Microbial lipid production using pure substrate (glucose and yeast extract) followed by conventional downstream process}

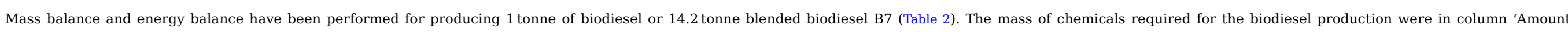

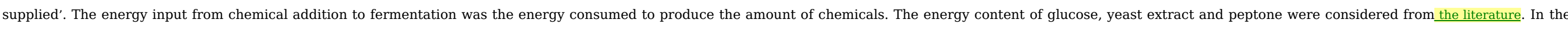

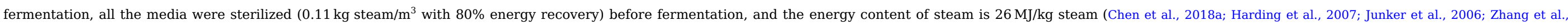

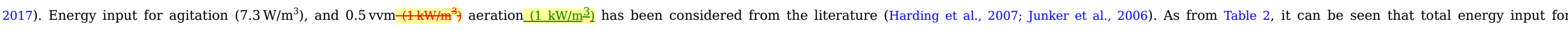

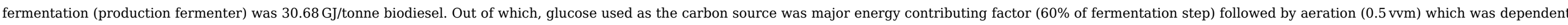

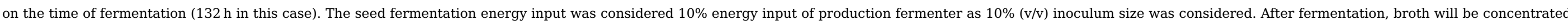

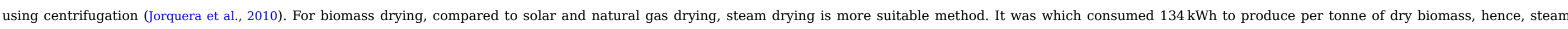

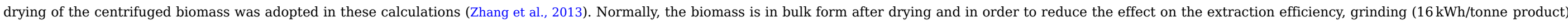

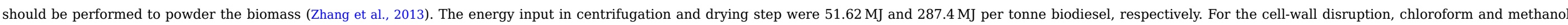

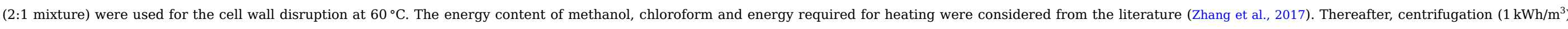

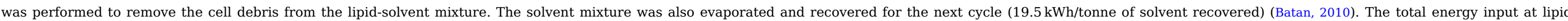

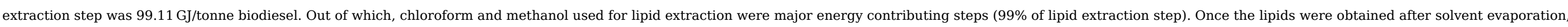

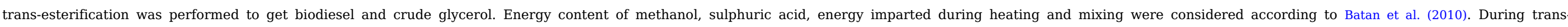

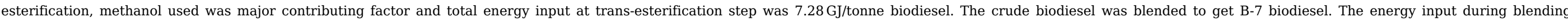

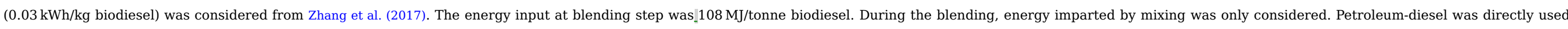

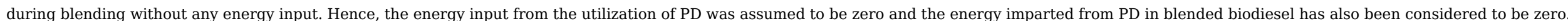

Table 2 Mass and energy balance of traditional biodiesel production process using microbial oil (glucose as substrate)

\begin{tabular}{|c|c|c|c|c|c|}
\hline Step & Items & Unit energy & Amount supplied & Energy input (MJ) & Energy input (\%) \\
\hline \multirow{7}{*}{ Production Fermentation (PF) } & \multicolumn{2}{|l|}{ Working volume } & $14.34 \mathrm{~m}^{3}$ & & \\
\hline & Sterilization (MJ/kg) & 26.00 & $1.58 \mathrm{~kg}$ & 41.01 & 0.03 \\
\hline & Yeast extract (MJ/kg) & 6.46 & $225 \mathrm{~kg}$ & 1453.50 & 1.03 \\
\hline & Peptone (MJ/kg) & 17.30 & $225 \mathrm{~kg}$ & 3892.50 & 2.77 \\
\hline & Glucose (MJ/kg) & 4.20 & $4363 \mathrm{~kg}$ & 18324.60 & 13.03 \\
\hline & Agitation $\left(\mathrm{W} / \mathrm{m}^{3}\right)$ & 7.30 & $14.34 \mathrm{~m}^{3}$ & 50.50 & 0.04 \\
\hline & Aeration $\left(\mathrm{kW} / \mathrm{m}^{3}\right)$ & 1.00 & $14.34 \mathrm{~m}^{3}$ & 6917.62 & 4.92 \\
\hline
\end{tabular}




\begin{tabular}{|c|c|c|c|c|c|}
\hline & & & & \multirow[b]{2}{*}{30679.73} & \multirow{3}{*}{$\begin{array}{l}21.82 \\
2.18\end{array}$} \\
\hline & \multicolumn{3}{|l|}{ Step energy input (MJ) } & & \\
\hline Seed fermentation & \multicolumn{3}{|c|}{$10 \%$ of Production fermenter (PFMJ) } & 3067.97 & \\
\hline \multirow{2}{*}{ Biomass Harvesting } & Centrifugation $\left(\mathrm{kWh} / \mathrm{m}^{3}\right)$ & 1.00 & $14.34 \mathrm{~m}^{3}$ & 51.62 & 0.04 \\
\hline & \multicolumn{3}{|l|}{ Step energy input (핖) } & 51.62 & 0.04 \\
\hline \multirow{3}{*}{ Biomass Drying } & Drying (kWh/tonne) & 134 & 1.5 tonne & 201 & 0.14 \\
\hline & Grinding (kWh/tonne) & 16 & 1.5 tonne & 86.4 & 0.06 \\
\hline & \multicolumn{3}{|l|}{ Step energy input (MJ) } & 287.4 & 0.2 \\
\hline \multirow{7}{*}{ Lipid extraction } & \multicolumn{2}{|l|}{ Reaction volume } & $7.63 \mathrm{~m}^{3}$ & & \\
\hline & Chloroform (MJ/kg) & 7.63 & $7577.56 \mathrm{~kg}$ & 57816.77 & 41.12 \\
\hline & Methanol (MJ/kg) & 20.00 & $2014.85 \mathrm{~kg}$ & 40296.96 & 28.66 \\
\hline & Solvent recovery $(\mathrm{k} w \mathrm{Wh} / \mathrm{t})$ & 19.50 & 9.6 tonne & 673.9 & 0.48 \\
\hline & Heating $\left(\mathrm{kW} / \mathrm{m}^{3}\right)$ & 2.72 & $7.63 \mathrm{~m}^{3}$ & 597.70 & 0.42 \\
\hline & Centrifugation $\left(\mathrm{kWh} / \mathrm{m}^{3}\right)$ & 1.00 & $7.63 \mathrm{~m}^{3}$ & 27.47 & 0.02 \\
\hline & \multicolumn{3}{|l|}{ Step energy input (MJ) } & 99113.97 & 70.5 \\
\hline \multirow{5}{*}{ Trans-esterification } & Methanol (MJ/kg) & 20.00 & $326.63 \mathrm{~kg}$ & 6532.60 & 4.64 \\
\hline & $\mathrm{NaOH}(\mathrm{MJ} / \mathrm{kg})$ & 18.50 & $21.96 \mathrm{~kg}$ & 406.26 & 0.29 \\
\hline & Mixing $(\mathrm{kWh} / \mathrm{kg}$ biodiesel $)$ & 0.03 & $1000 \mathrm{~kg}$ & 108.00 & 0.08 \\
\hline & Heating (kJ/kg biodiesel) & 240.00 & $1000 \mathrm{~kg}$ & 240.00 & 0.17 \\
\hline & \multicolumn{3}{|l|}{ Step energy input (MJ) } & 7286.86 & 5.18 \\
\hline \multirow[t]{2}{*}{ Blending } & Mixing $(\mathrm{kWh} / \mathrm{kg}$ biodiesel $)$ & 0.03 & $1000 \mathrm{~kg}$ & 108.00 & 0.08 \\
\hline & \multicolumn{3}{|l|}{ Step energy input (MJ) } & 108.00 & 0.08 \\
\hline \multicolumn{4}{|l|}{ Total energy input (MJ) } & \multicolumn{2}{|l|}{140595.5} \\
\hline \multicolumn{4}{|l|}{ Total energy input (GJ) } & \multicolumn{2}{|l|}{140.6} \\
\hline \multicolumn{4}{|l|}{ Credits - crude glycerol (GJ) } & \multicolumn{2}{|l|}{3.85} \\
\hline \multicolumn{4}{|l|}{ Net energy input (GJ) } & \multicolumn{2}{|l|}{136.75} \\
\hline \multicolumn{4}{|l|}{ Net energy output (GJ) } & \multicolumn{2}{|l|}{37.80} \\
\hline \multicolumn{4}{|l|}{ Energy gain (GJ) } & \multicolumn{2}{|l|}{-98.95} \\
\hline \multicolumn{4}{|l|}{ Energy conversion efficiency } & 0.28 & \\
\hline
\end{tabular}

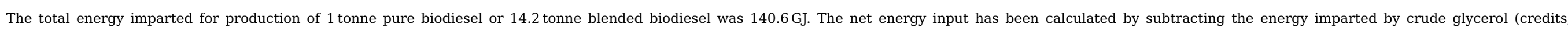

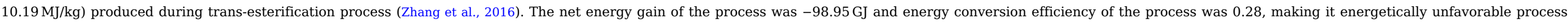

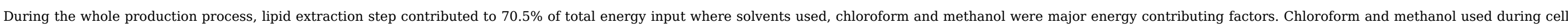

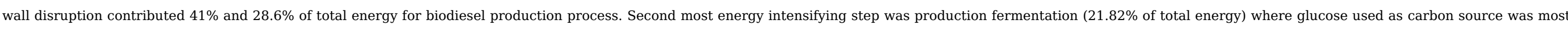

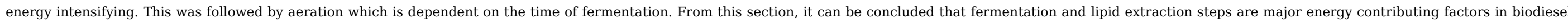




\subsection{Microbial lipid production using pure substrate (glucose and yeast extract) followed by INRS downstream process}

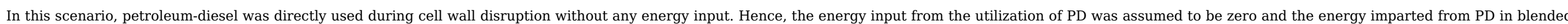

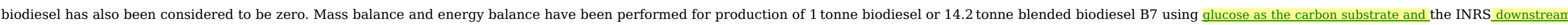

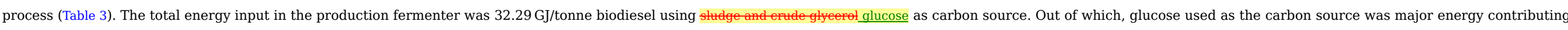

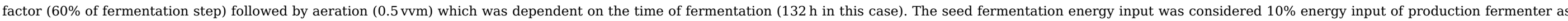
$10 \%(\mathrm{v} / \mathrm{v})$ inoculum size was considered.

Table 3 Mass and energy balance of developedINRS biodiesel production process using microbial oil (glucose as substrate)

\begin{tabular}{|c|c|c|c|c|c|}
\hline Step & Items & Unit energy input & Amount supplied & Total energy input (MJ) & Energy input (\%) \\
\hline \multirow{8}{*}{ Production Fermentation (PF) } & \multicolumn{2}{|l|}{ Reaction volume } & $15.09 \mathrm{~m}^{3}$ & & \\
\hline & Sterilization (MJ/kg) & 26.00 & $1.66 \mathrm{~kg}$ & 43.16 & 0.11 \\
\hline & yeast extract (MJ/kg) & 6.46 & $236.90 \mathrm{~kg}$ & 1530.37 & 3.21 \\
\hline & peptone (MJ/kg) & 17.30 & $236.90 \mathrm{~kg}$ & 4098.37 & 8.59 \\
\hline & Glucose (MJ/kg) & 4.20 & $4591.70 \mathrm{~kg}$ & 19285.14 & 40.41 \\
\hline & Agitation $\left(\mathrm{W} / \mathrm{m}^{3}\right)$ & 7.30 & $15.09 \mathrm{~m}^{3}$ & 53.14 & 0.11 \\
\hline & Aeration $\left(\mathrm{KW} / \mathrm{m}^{3}\right)$ & 1.00 & $15.09 \mathrm{~m}^{3}$ & 7279.42 & 15.25 \\
\hline & \multicolumn{3}{|l|}{ Step energy input (MJ) } & 32289.6 & 67.66 \\
\hline Seed fermentation & \multicolumn{3}{|c|}{$10 \%$ of Production fermenter (PFMI) } & 3228.96 & 6.77 \\
\hline \multirow{3}{*}{ Biomass settling } & EPS (MJ/kg) & 18.84 & $191.24 \mathrm{~kg}$ & 3602.96 & 7.55 \\
\hline & $\mathrm{CaCl} 2(\mathrm{MJ} / \mathrm{kg})$ & 7.20 & $117.25 \mathrm{~kg}$ & 844.20 & 1.77 \\
\hline & \multicolumn{3}{|l|}{ Step energy input ( $\mathrm{PF} \underline{\mathrm{MJ}}$ ) } & 4447.16 & 9.32 \\
\hline \multirow{6}{*}{ Lipid extraction } & nN-LS (MJ/kg) & 5.76 & $64.28 \mathrm{~kg}$ & 370.25 & 0.78 \\
\hline & Petro-diesel (MJ/kg) & 0.00 & $13239.54 \mathrm{~kg}$ & 0.00 & 0.00 \\
\hline & Agitation $\left(\mathrm{W} / \mathrm{m}^{3}\right)$ & 7.30 & $16.63 \mathrm{~m}^{3}$ & 0.31 & 0.00 \\
\hline & Heating $\left(\mathrm{kW} / \mathrm{m}^{3}\right)$ & 2.72 & $16.63 \mathrm{~m}^{3}$ & 40.71 & 0.09 \\
\hline & Centrifugation $\left(\mathrm{kWh} / \mathrm{m}^{3}\right)$ & 1.00 & $16.63 \mathrm{~m}^{3}$ & 59.87 & 0.13 \\
\hline & \multicolumn{3}{|l|}{ Step energy input (MJ) } & 471.14 & 0.99 \\
\hline \multirow{5}{*}{ Trans-esterification } & Methanol (kg) & 20.00 & $326.58 \mathrm{~kg}$ & 6531.60 & 13.69 \\
\hline & $\mathrm{NaOH}(\mathrm{kg})$ & 18.50 & $21.96 \mathrm{~kg}$ & 406.26 & 0.85 \\
\hline & Mixing (kWh/kg biodiesel) & 0.03 & $1000 \mathrm{~kg}$ & 108.00 & 0.23 \\
\hline & Heating (kJ/kg biodiesel) & 240.00 & $1000 \mathrm{~kg}$ & 240.00 & 0.5 \\
\hline & \multicolumn{3}{|l|}{ Step energy input (MJ) } & 7285.86 & 15.27 \\
\hline \multicolumn{4}{|l|}{ Total energy input (MJ) } & \multicolumn{2}{|l|}{47722.72} \\
\hline
\end{tabular}




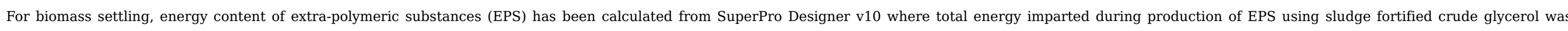

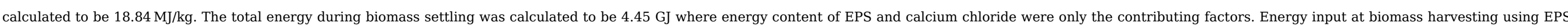
and $\mathrm{CaCl}_{2}$ was higher than centrifugation (Table 2). Hence two cases have been considered in biomass harvesting: a) centrifuge aided biomass harvesting and b) EPS aided biomass settling.

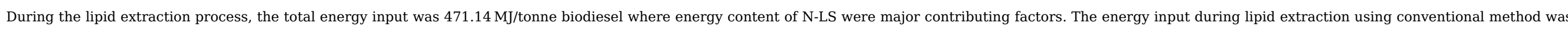

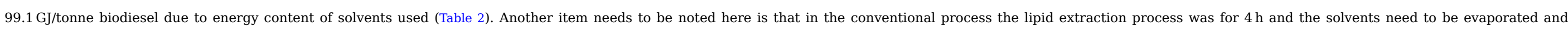

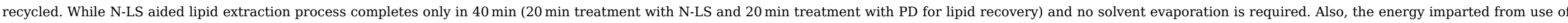

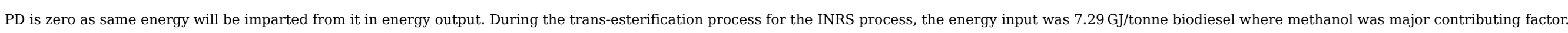

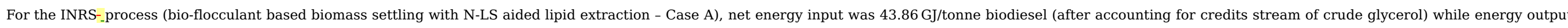

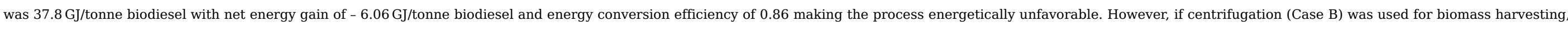

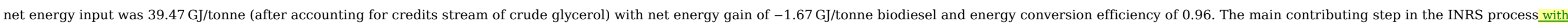

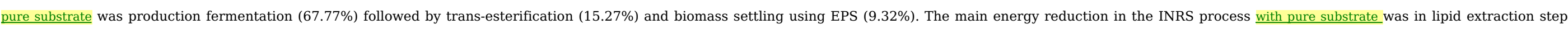

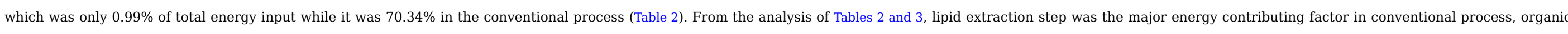

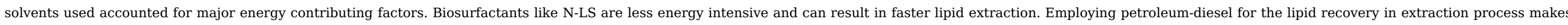

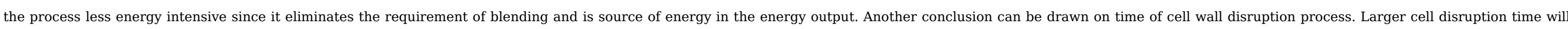

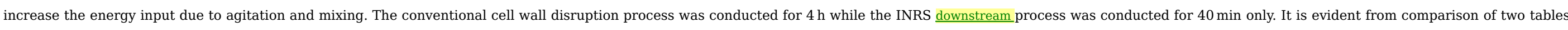

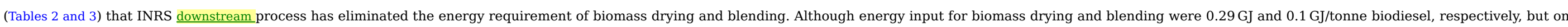
a higher scale of production, they can be quite energy intensive.

\subsection{Microbial lipid production using sludge fortified with crude glycerol media followed by INRS downstream process}

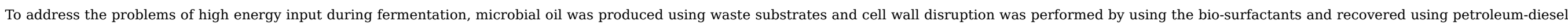
The following sub-section discusses the mass and energy balance of the INRS process.

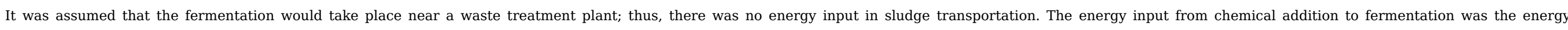

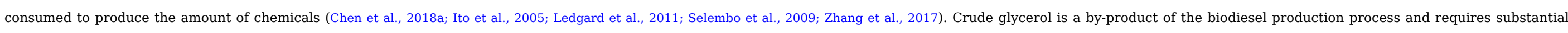

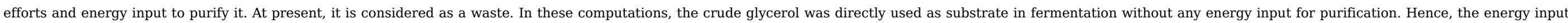




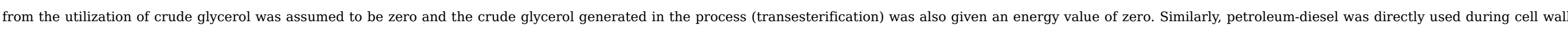

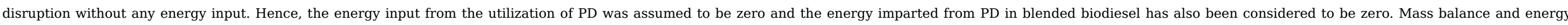

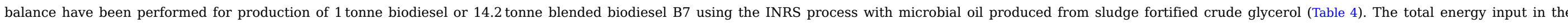

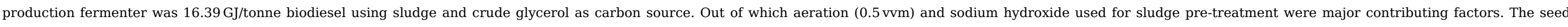

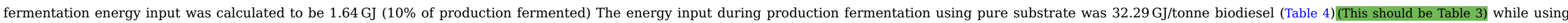
waste substrate it was $16.39 \mathrm{GJ} /$ tonne biodiesel only indicating that on employing waste substrates, fermentation energy input has been reduced to $50 \%$.

Table 4 Mass and energy balance of developedINRS biodiesel production process using microbial oil (sludge fortified crude glycerol as substrate).

\begin{tabular}{|c|c|c|c|c|c|}
\hline Step & Items & Unit energy & Amount supplied & Energy input (MJ) & Energy input (\%) \\
\hline \multirow{7}{*}{ Production Fermentation (PF) } & \multicolumn{2}{|l|}{ Working volume } & $64.5 \mathrm{~m}^{3}$ & & \\
\hline & Sterilization (MJ/kg) & 26.00 & $7.10 \mathrm{~kg}$ & 184.47 & 0.61 \\
\hline & $\mathrm{NaOH}(\mathrm{MJ} / \mathrm{kg})$ & 18.50 & $186 \mathrm{~kg}$ & 3441.00 & 11.34 \\
\hline & $\mathrm{H}_{2} \mathrm{SO}_{4}(\mathrm{MJ} / \mathrm{kg})$ & 7.10 & $216 \mathrm{~kg}$ & 1533.60 & 5.04 \\
\hline & Agitation $\left(\mathrm{W} / \mathrm{m}^{3}\right)$ & 7.30 & $64.50 \mathrm{~m}^{3}$ & 81.36 & 0.27 \\
\hline & Aeration $\left(\mathrm{kW} / \mathrm{m}^{3}\right)$ & 1.00 & $64.50 \mathrm{~m}^{3}$ & 11145.60 & 36.74 \\
\hline & \multicolumn{3}{|l|}{ Step energy input (MJ) } & 16386.03 & 54 \\
\hline Seed fermentation & \multicolumn{3}{|c|}{$10 \%$ of Production fermenter ( $P F \underline{M}$ J) } & 1638.60 & 5.4 \\
\hline \multirow{3}{*}{ Biomass settling } & EPS (MJ/kg) & 18.84 & $114.76 \mathrm{~kg}$ & 2162.08 & 7.12 \\
\hline & $\mathrm{CaCl}_{2}(\mathrm{MJ} / \mathrm{kg})$ & 7.20 & $367 \mathrm{~kg}$ & 2642.40 & 8.71 \\
\hline & \multicolumn{3}{|l|}{ Step energy input (PFMJ) } & 4804.48 & 15.83 \\
\hline \multirow{8}{*}{ Lipid extraction } & \multicolumn{2}{|l|}{ Working volume } & $14.93 \mathrm{~m}^{3}$ & & \\
\hline & N-LS (MJ/kg) & 5.76 & $57.38 \mathrm{~kg}$ & 330.51 & 1.09 \\
\hline & FNA (MJ/kg) & 3.20 & $26.69 \mathrm{~kg}$ & 85.41 & 0.28 \\
\hline & Petro-diesel (MJ/kg) & 0 & $13239.54 \mathrm{~kg}$ & 0.00 & 0.00 \\
\hline & Agitation $\left(\mathrm{W} / \mathrm{m}^{3}\right)$ & 7.3 & $14.93 \mathrm{~m}^{3}$ & 0.27 & 0.00 \\
\hline & Heating $\left(\mathrm{kW} / \mathrm{m}^{3}\right)$ & 2.72 & $14.93 \mathrm{~m}^{3}$ & 36.55 & 0.16 \\
\hline & Centrifugation $\left(\mathrm{kWh} / \mathrm{m}^{3}\right)$ & 1.00 & $14.93 \mathrm{~m}^{3}$ & 53.75 & 0.18 \\
\hline & \multicolumn{3}{|l|}{ Step energy input (MJ) } & 506.49 & 1.67 \\
\hline \multirow{5}{*}{ Trans-esterification } & Methanol (kg) & 20.00 & $326.5 \mathrm{~kg}$ & 6530.00 & 21.52 \\
\hline & Sulphuric acid (kg) & 7.10 & $19 \mathrm{~kg}$ & 134.90 & 0.44 \\
\hline & Mixing (kWh/kg biodiesel) & 0.03 & $1000 \mathrm{~kg}$ & 108.00 & 0.36 \\
\hline & Heating (kJ/kg biodiesel) & 240.00 & $1000 \mathrm{~kg}$ & 240.00 & 0.79 \\
\hline & \multicolumn{3}{|l|}{ Step energy input (MJ) } & 7012.90 & 23.11 \\
\hline \multicolumn{4}{|l|}{ Total energy input (MJ) } & \multicolumn{2}{|l|}{30348.5} \\
\hline
\end{tabular}




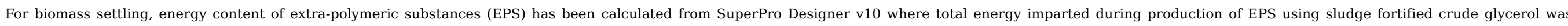

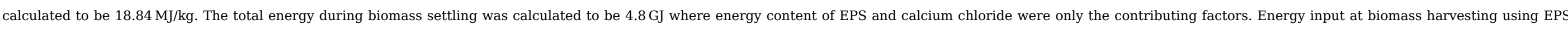
and $\mathrm{CaCl}_{2}$ was higher than centrifugation (Table 3). Hence two cases have been considered in biomass harvesting: a) centrifuge aided biomass harvesting and b) EPS aided biomass settling.

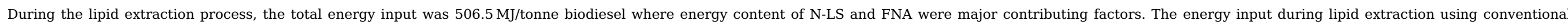

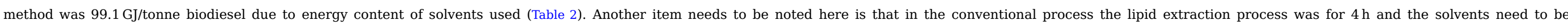

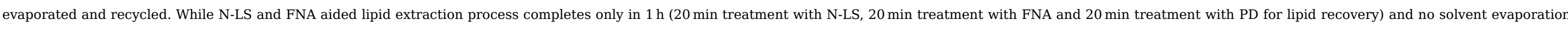

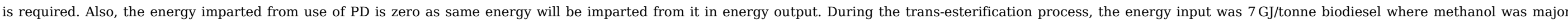

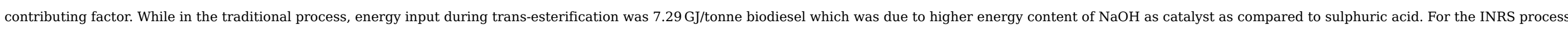

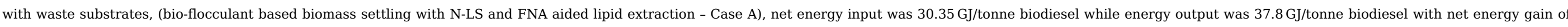

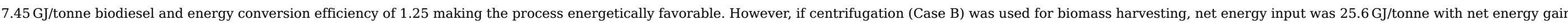

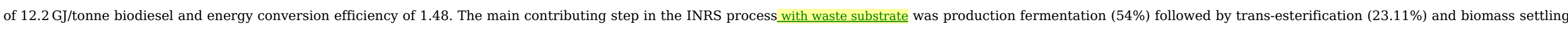
using EPS (15.83\%). The main energy reduction in the INRS process was in lipid extraction step, which was only $1.67 \%$ of total energy input while it was $70.34 \%$ in the conventional process.

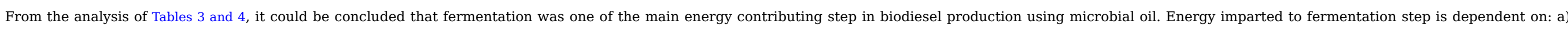

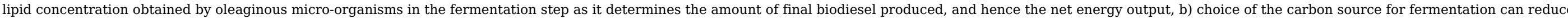

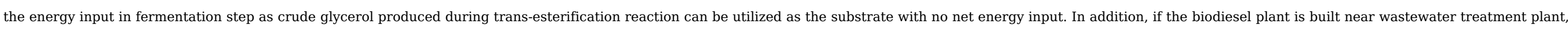

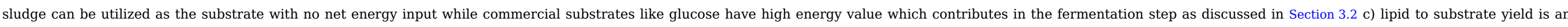

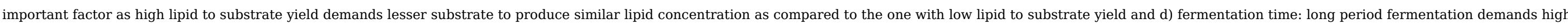
electricity consumption, which increases energy input during agitation and aeration.

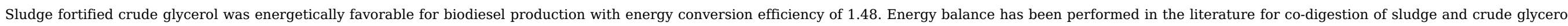

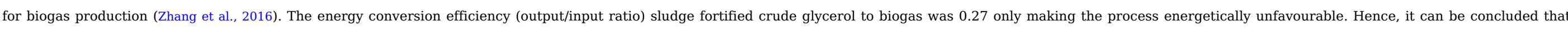
utilizing sludge and crude glycerol for biodiesel production would be 5.4 times more energetically favorable than co-digesting them for biogas production.

\subsection{Microbial lipid production using crude glycerol media followed by INRS downstream process}

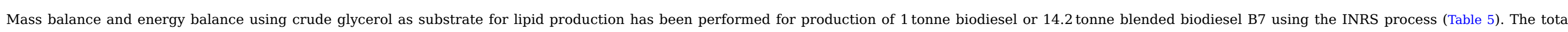

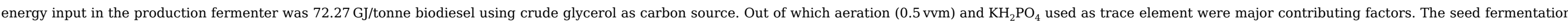

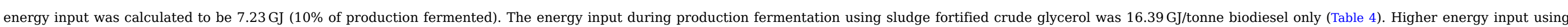

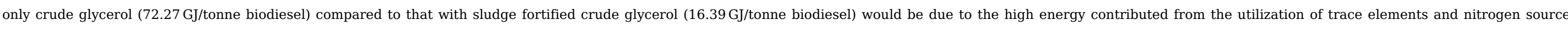

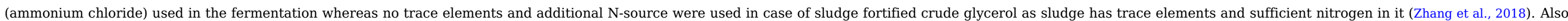


Table 5 Mass and energy balance of developedINRS biodiesel production process using microbial oil (crude glycerol as substrate).

\begin{tabular}{|c|c|c|c|c|c|}
\hline Step & Items & Unit energy input & Amount supplied & Total energy input (MJ) & Energy input (\%) \\
\hline \multirow{9}{*}{ Production Fermentation (PF) } & \multicolumn{2}{|l|}{ Reaction volume } & $192.24 \mathrm{~m}^{3}$ & & \\
\hline & Sterilization (MJ/kg) & 26.00 & $21.15 \mathrm{~kg}$ & 549.81 & 0.59 \\
\hline & $\mathrm{H}_{3} \mathrm{PO}_{4}(\mathrm{MJ} / \mathrm{kg})$ & 5.30 & $1414.50 \mathrm{~kg}$ & 7496.85 & 8.05 \\
\hline & $\mathrm{KH}_{2} \mathrm{PO}_{4}(\mathrm{MJ} / \mathrm{kg})$ & 10.30 & $969.84 \mathrm{~kg}$ & 9989.35 & 10.72 \\
\hline & $\mathrm{Na}_{2} \mathrm{HPO}_{4}(\mathrm{MJ} / \mathrm{kg})$ & 8.21 & $341.24 \mathrm{~kg}$ & 2801.58 & 3.01 \\
\hline & $\mathrm{NH}_{4} \mathrm{Cl}(\mathrm{MJ} / \mathrm{kg})$ & 8.64 & $143.68 \mathrm{~kg}$ & 1241.40 & 1.33 \\
\hline & Agitation $\left(\mathrm{W} / \mathrm{m}^{3}\right)$ & 7.30 & $192.24 \mathrm{~m}^{3}$ & 363.75 & 0.39 \\
\hline & Aeration $\left(\mathrm{kW} / \mathrm{m}^{3}\right)$ & 1.00 & $192.24 \mathrm{~m}^{3}$ & 49828.61 & 53.47 \\
\hline & \multicolumn{3}{|l|}{ Step energy input (MJ) } & 72271.34 & 77.56 \\
\hline Seed fermentation & \multicolumn{3}{|c|}{$10 \%$ of Production fermenter (PFMI) } & 7227.13 & 7.76 \\
\hline \multirow{3}{*}{ Biomass settling } & EPS (MJ/kg) & 18.84 & $13.25 \mathrm{~kg}$ & 249.63 & 0.27 \\
\hline & $\mathrm{CaCl}_{2}(\mathrm{MJ} / \mathrm{kg})$ & 7.20 & $768.20 \mathrm{~kg}$ & 5531.04 & 5.94 \\
\hline & \multicolumn{3}{|l|}{ Step energy input (PFMJ) } & 5780.67 & 6.21 \\
\hline \multirow{6}{*}{ Lipid extraction } & N-LS (MJ/kg) & 5.76 & $91.38 \mathrm{~kg}$ & 526.35 & 0.56 \\
\hline & Petro-diesel (MJ/kg) & 0 & $13239.54 \mathrm{~kg}$ & 0.00 & 0.00 \\
\hline & Agitation $\left(\mathrm{W} / \mathrm{m}^{3}\right)$ & 7.30 & $15.55 \mathrm{~m}^{3}$ & 0.29 & 0.00 \\
\hline & Heating $\left(\mathrm{kW} / \mathrm{m}^{3}\right)$ & 2.72 & $15.55 \mathrm{~m}^{3}$ & 38.07 & 0.04 \\
\hline & Centrifugation $\left(\mathrm{kWh} / \mathrm{m}^{3}\right)$ & 1.00 & $15.55 \mathrm{~m}^{3}$ & 55.98 & 0.06 \\
\hline & \multicolumn{3}{|l|}{ Step energy input (MJ) } & 620.68 & 0.67 \\
\hline \multirow{5}{*}{ Trans-esterification } & Methanol (MJ/kg) & 20.00 & $326.58 \mathrm{~kg}$ & 6531.60 & 7.01 \\
\hline & $\mathrm{NaOH}(\mathrm{MJ} / \mathrm{kg})$ & 18.50 & $21.96 \mathrm{~kg}$ & 406.26 & 0.44 \\
\hline & Mixing (kWh/kg biodiesel) & 0.03 & $1000 \mathrm{~kg}$ & 108.00 & 0.12 \\
\hline & Heating (kJ/kg biodiesel) & 240.00 & $1000 \mathrm{~kg}$ & 240.00 & 0.26 \\
\hline & \multicolumn{3}{|l|}{ Step energy input (MJ) } & 7285.86 & 7.82 \\
\hline \multicolumn{4}{|l|}{ NetTotal energy input (MJ) } & \multicolumn{2}{|l|}{93185.69} \\
\hline \multicolumn{4}{|l|}{ Net energy input (GJ) - Case A } & \multicolumn{2}{|l|}{93.19} \\
\hline \multicolumn{4}{|l|}{ Net energy input (GJ) - Case B } & \multicolumn{2}{|l|}{$\mathbf{8 8 . 1}$} \\
\hline \multicolumn{4}{|l|}{ Net energy output (GJ) } & \multicolumn{2}{|l|}{37.80} \\
\hline \multicolumn{4}{|l|}{ Energy gain (GJ) - Case A } & \multicolumn{2}{|l|}{-55.39} \\
\hline \multicolumn{4}{|l|}{ Energy gain (GJ) - Case A } & \multicolumn{2}{|l|}{-50.30} \\
\hline
\end{tabular}


Note: Case A is for bio-flocculant based biomass settling while Case B is for centrifuge aided biomass harvesting.

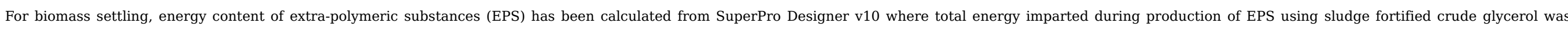

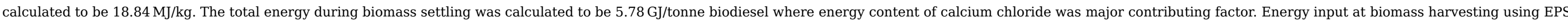
and $\mathrm{CaCl}_{2}$ was higher than centrifugation. Hence two cases have been considered in biomass harvesting: a) centrifuge aided biomass harvesting and b) EPS aided biomass settling.

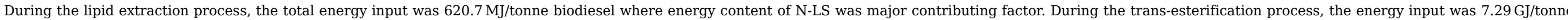

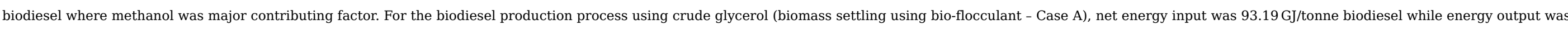

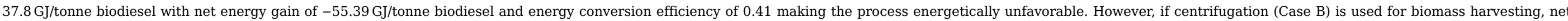

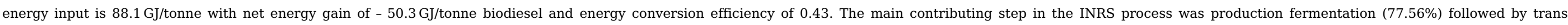

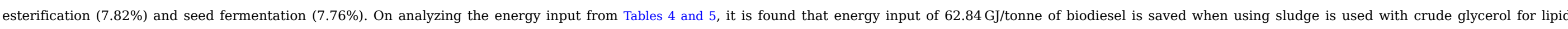
production which is the additional energy required for the nutrients and trace elements during fermentation.

\subsection{Direct oil extraction from scum and conversion to biodiesel}

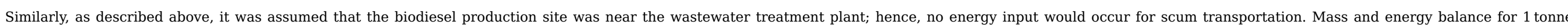

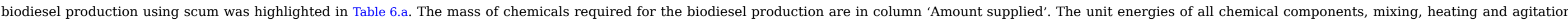

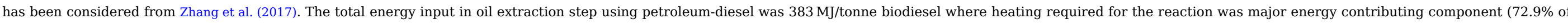

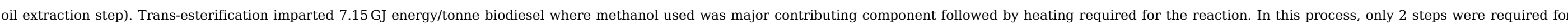

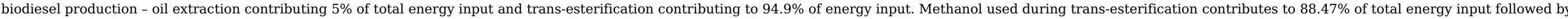

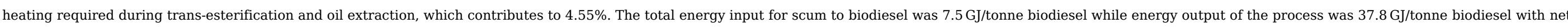

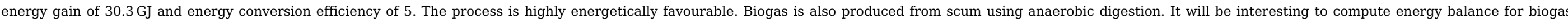
production from scum and compare with biodiesel production from scum.

Table 6.a Mass and energy balance of biodiesel production process using scum.

\begin{tabular}{|c|c|c|c|c|c|}
\hline Step & Items & Unit energy & Amount supplied & Energy input (MJ) & Energy input (\%) \\
\hline \multirow{7}{*}{ Oil extraction } & \multicolumn{2}{|l|}{ Scum used } & $11 \mathrm{~m}^{3}$ & & \\
\hline & \multicolumn{2}{|c|}{ Reaction volume (including PD) } & $28.56 \mathrm{~m}^{3}$ & & \\
\hline & Petro-diesel (MJ/kg) & 0 & $13971 \mathrm{~kg}$ & 0.00 & 0.00 \\
\hline & Agitation $\left(\mathrm{W} / \mathrm{m}^{3}\right)$ & 7.30 & $28.56 \mathrm{~m}^{3}$ & 0.75 & 0.01 \\
\hline & Heating $\left(\mathrm{kW} / \mathrm{m}^{3}\right)$ & 2.72 & $28.56 \mathrm{~m}^{3}$ & 279.7 & 3.71 \\
\hline & Centrifugation $\left(\mathrm{kWh} / \mathrm{m}^{3}\right)$ & 1.00 & $28.56 \mathrm{~m}^{3}$ & 102.8 & 1.36 \\
\hline & \multicolumn{3}{|l|}{ Step energy input (MJ) } & 383.2 & 5.08 \\
\hline \multirow[b]{3}{*}{ Trans-esterification } & Methanol (MJ/kg) & 20 & $333.40 \mathrm{~kg}$ & 6668 & 88.97 \\
\hline & Sulphuric acid (MJ/kg) & 7.10 & $19.37 \mathrm{~kg}$ & 137.53 & 1.82 \\
\hline & Mixing (kWh/kg biodiesel) & 0.03 & $1000 \mathrm{~kg}$ & 108.00 & 1.43 \\
\hline
\end{tabular}

$19.37 \mathrm{~kg}$

108.00 


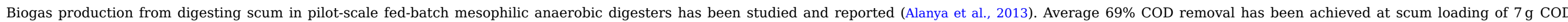

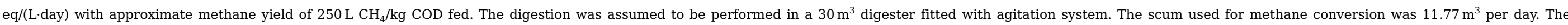

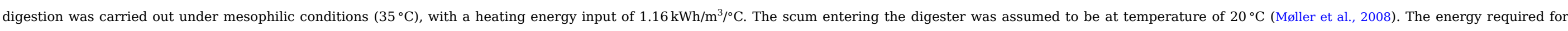

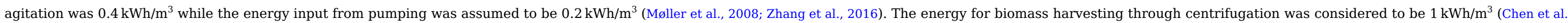

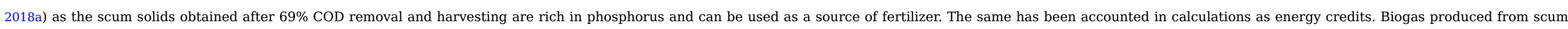

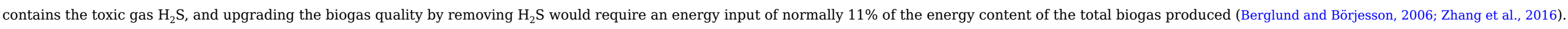

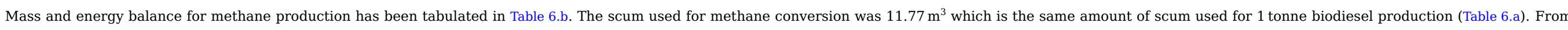

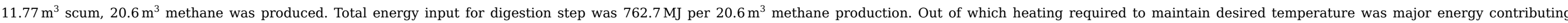

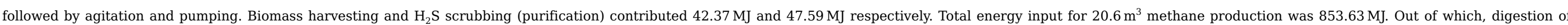

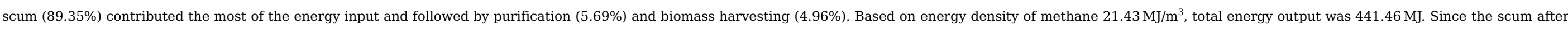

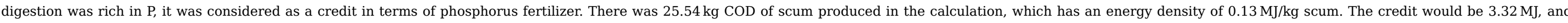

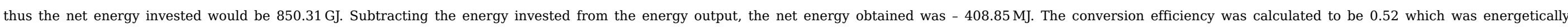
unfavourable. To compare the Tables 6.a and 6.b, it suggests that scum to biodiesel was 9.6 times more energetically favorable than scum to methane using same amount of scum for both the process.

Table 6.b Mass and energy balance for methane production using scum.

\begin{tabular}{|c|c|c|c|c|c|}
\hline Step & Items & Unit energy input & Amount supplied & Total energy input (MJ) & Energy input (\%) \\
\hline \multirow{6}{*}{ Digestion } & \multicolumn{2}{|l|}{ Scum used } & $11.77 \mathrm{~m}^{3}$ & & \multirow[b]{2}{*}{0.00} \\
\hline & Scum (MJ/kg) & 0.00 & $82.39 \mathrm{~kg}$ COD & 0.00 & \\
\hline & Agitation $\left(\mathrm{kWh} / \mathrm{m}^{3}\right)$ & 0.40 & $11.77 \mathrm{~m}^{3}$ & 16.95 & 1.99 \\
\hline & Pumping $\left(\mathrm{kWh} / \mathrm{m}^{3}\right)$ & 0.20 & $11.77 \mathrm{~m}^{3}$ & 8.47 & 0.99 \\
\hline & Heating $\left(\mathrm{kWh} / \mathrm{m}^{3}\right)$ & 1.16 & $11.77 \mathrm{~m}^{3}$ & 737.27 & 86.37 \\
\hline & \multicolumn{3}{|l|}{ Step energy input_(MJ) } & 762.7 & 89.35 \\
\hline Biomass harvesting & Centrifugation $\left(\mathrm{kWh} / \mathrm{m}^{3}\right)$ & 1.00 & $11.77 \mathrm{~m}^{3}$ & 42.372 & 4.96 \\
\hline Purification & \multicolumn{3}{|c|}{ Purification ( $11 \%$ of energy output/methane yield) } & 47.56 & 5.69 \\
\hline \multicolumn{4}{|l|}{ Total energy Input (MJ) } & \multicolumn{2}{|l|}{852.65} \\
\hline \multicolumn{4}{|l|}{ Total energy Input (GJ) } & \multicolumn{2}{|l|}{0.85} \\
\hline \multicolumn{4}{|l|}{ Credits (MJ) } & \multicolumn{2}{|l|}{3.32} \\
\hline \multicolumn{4}{|l|}{ Net energy input (GJ) } & \multicolumn{2}{|l|}{0.85} \\
\hline
\end{tabular}




\section{Conclusion}

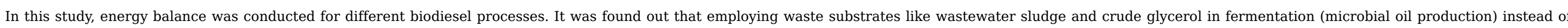

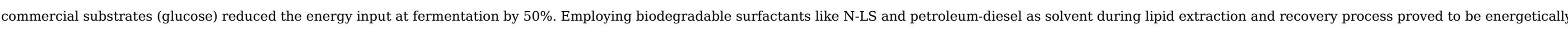

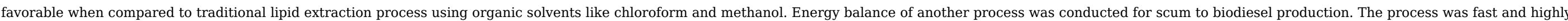
energetically favorable.

\section{Acknowledgements}

Authors would like to acknowledge the Natural Sciences and Engineering Research Council of Canada (grant A4984, Strategic grant 412994-11, Canada Research Chair) for financial support.

\section{References}

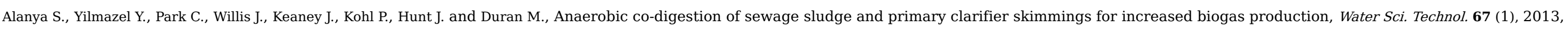
174-179.

Apostolakou A., Kookos I., Marazioti C. and Angelopoulos K., Techno-economic analysis of a biodiesel production process from vegetable oils, Fuel Process. Technol. 90 (7), 2009, 1023-1031.

Batan L., Quinn J., Willson B. and Bradley T., Net energy and greenhouse gas emission evaluation of biodiesel derived from microalgae, Environ. Sci. Technol. 44 (20), 2010, 7975-7980.

Berglund M. and Börjesson P., Assessment of energy performance in the life-cycle of biogas production, Biomass Bioenergy 30 (3), $2006,254-266$.

Bi C.-H., Min M., Nie Y., Xie Q.-L., Lu Q., Deng X.-Y., Anderson E., Li D., Chen P. and Ruan R., Process development for scum to biodiesel conversion, Bioresour. Technol. 185, 2015, 185-193.

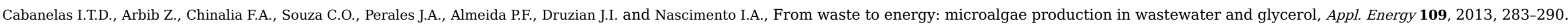

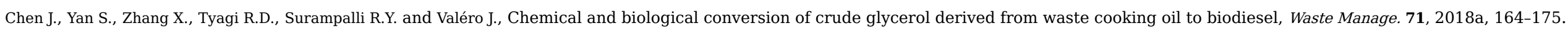

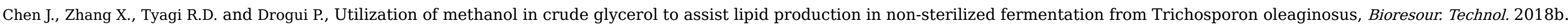

Chen J., Zhang X., Yan S., Tyagi R.D. and Drogui P., Lipid production from fed-batch fermentation of crude glycerol directed by the kinetic study of batch fermentations, Fuel 209, 2017, 1-9.

Gouda M.K., Omar S.H. and Aouad L.M., Single cell oil production by Gordonia sp. DG using agro-industrial wastes, World J. Microbiol. Biotechnol. 24 (9), $2008,1703$.

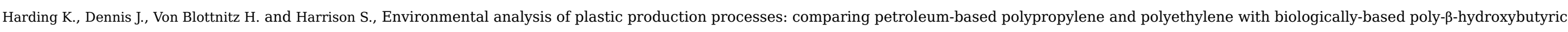
acid using life cycle analysis, J. Biotechnol. 130 (1), 2007, 57-66.

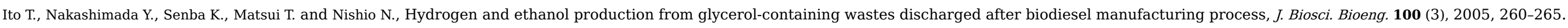

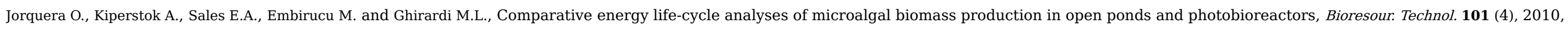

$1406-1413$.

Junker B., Lester M., Brix T., Wong D. and Nuechterlein J., A next generation, pilot-scale continuous sterilization system for fermentation media, Bioprocess Biosyst. Eng. 28 (6), 2006, 351-378.

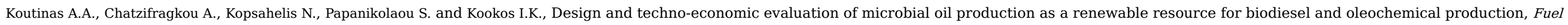
116, 2014, 566-577. 
Ledgard S.F., Boyes M. and Brentrup F., Life Cycle Assessment of Local and Imported Fertilisers used on New Zealand Farms, 2011, Ministry of Agriculture and Forestry.

Liang M.-H. and Jiang J.-G., Advancing oleaginous microorganisms to produce lipid via metabolic engineering technology, Prog. Lipid Res. 52 (4), 2013, 395-408.

Liang Y., Cui Y., Trushenski J. and Blackburn J.W., Converting crude glycerol derived from yellow grease to lipids through yeast fermentation, Bioresour. Technol. 101 (19), 2010, 7581-7586.

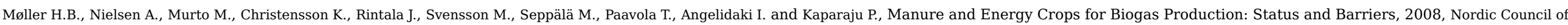
Ministers; Copenhagen, Denmark.

Pimentel D. and Patzek T.W., Ethanol production using corn, switchgrass, and wood; biodiesel production using soybean and sunflower, Nat. Resour. Res. 14 (1), 2005, 65-76.

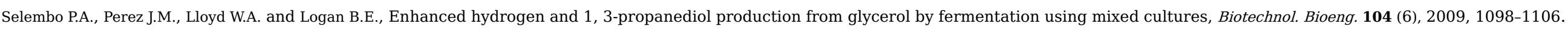

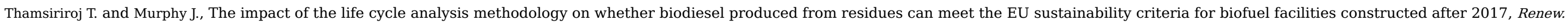
Energy 36 (1), 2011, 50-63.

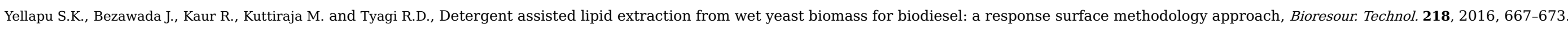
Yellapu S.K., Kaur R., Kumar L.R., Tiwari B., Zhang X. and Tyagi R.D., Recent developments of downstream processing for microbial lipids and conversion to biodiesel, Bioresour. Technol. 2018a.

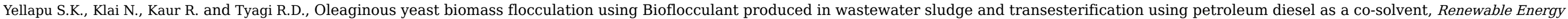
2018b.

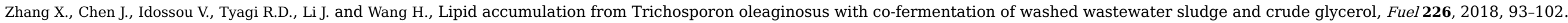

Zhang X., Chen J., Yan S., Tyagi R.D., Surampalli R.Y. and Li J., Lipid production for biodiesel from sludge and crude glycerol, Water Environ. Res. 89 (5), 2017 , $424-439$.

Zhang X., Yan S., Tyagi R.D., Surampalli R. and Valéro J.R., Wastewater sludge as raw material for microbial oils production, Appl. Energy 135, 2014 , $192-201$.

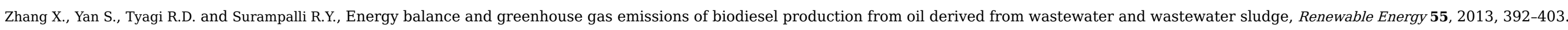

Zhang X., Yan S., Tyagi R.D., Surampalli R.Y. and Valéro J.R., Energy balance of biofuel production from biological conversion of crude glycerol, J. Environ. Manage. 170, 2016, 169-176.

\section{Graphical abstract}

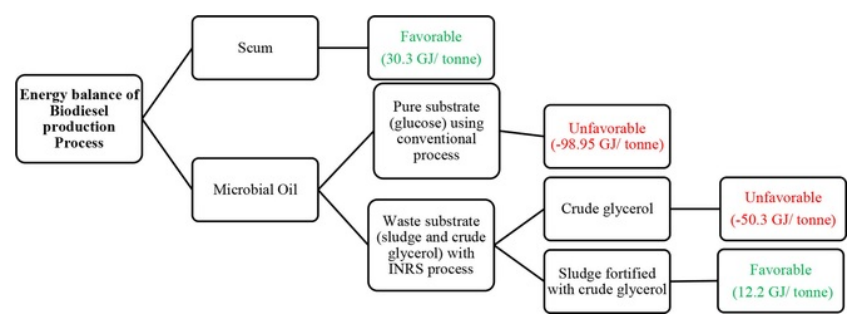

\section{Highlights}

- Biodiesel production from scum is more favorable than methane production. 
- Sludge fortified crude glycerol reduces energy input at fermentation for microbial lipid production

- Lipid extraction using N-LS and Petroleum diesel is quick and less energy intensive.

\section{Queries and Answers}

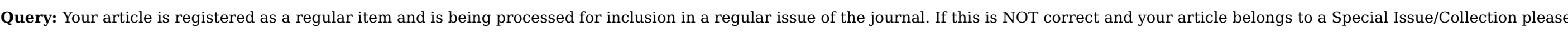
contact l.daniel@elsevier.com immediately prior to returning your corrections.

Answer: Yes, a regular issue

Query: The author names have been tagged as given names and surnames (surnames are highlighted in teal color). Please confirm if they have been identified correctly. Answer: Yes

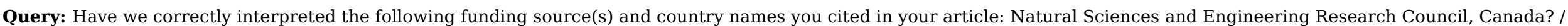
Answer: Yes

Query: Please check the layout of Tables 2 to 5, and correct if necessary Answer: Table layout is correct. Small changes have been incorporated. 